Onkologe 2014 · 20:530-532

DOI 10.1007/s00761-013-2523-z

Online publiziert: 16. Mai 2014

(c) Springer-Verlag Berlin Heidelberg 2014

\section{Schadendorf ${ }^{1}$ P.M. Schlag ${ }^{2}$}

${ }^{1}$ Klinik für Dermatologie, Universitätsklinikum Essen, Universität Duisburg-Essen

${ }^{2}$ Berlin

\title{
Malignes Melanom
}

Die Behandlung des malignen Melanoms wurde lange als frustrierend betrachtet, und seine Therapieresistenz insbesondere gegenüber chemotherapeutischen Behandlungsansätzen machte es oftmals zur letzten Station einer erfolglosen Medikamentenentwicklung. Das hat sich in den letzten 5 Jahren verändert. Diese Änderungen schlagen sich in vielen Bereichen nieder und sind nicht nur auf die Systemtherapie im Stadium IV begrenzt.

Jährlich werden mehr als 20.000 Melanome neu in Deutschland diagnostiziert. Nahezu $80 \%$ davon als Melanoma in situ oder mit einer prognostisch sehr guten, geringen Tumoreindringtiefe von $<1 \mathrm{~mm}$, bei der nur in Ausnahmefällen mit einer Progression zu rechnen ist. In einer interdisziplinären Zusammenschau werden in diesem Heft die aktuellen Entwicklungen und Strategien in Prävention, Diagnostik und Therapie beleuchtet und bewertet.

\section{》) Die Einführung des gesetzlichen Hautkrebsscreenings ist ein wichtiger Fortschritt der letzten 5 Jahre}

Neben dem gesetzlichen Besuchsverbot der Solarien von Jugendlichen unter 18 Jahren ist die Einführung des gesetzlichen Hautkrebsscreenings eine wesentliche Errungenschaft der letzten 5 Jahre. M. Augustin und A. Katalinic fassen die hierauf basierenden aktuellen epidemiologischen Daten am Beispiel des epidemiologischen Krebsregisters SchleswigHolstein zusammen, die u. a. auch darauf hindeuten, dass die Melanommortalität auf diese Weise gesenkt werden kann. Die Standards von Diagnostik und Therapie des Melanoms sind in der kürzlich publizierten S3-Leitlinie definiert worden, die auch alle relevanten Diagnostik- und Therapiealgorithmen enthält [1]. Sie stellt auch die Richtschnur dar, an der sich E. Dippel et al. bei der Zusammenfassung der chirurgischen Prinzipien und deren differenziertem Einsatz ausrichten.

In der adjuvanten Therapie, dargestellt von M. Fluck und C. Garbe, dominiert weiterhin Interferon, dessen Anwendung aber zunehmend abnimmt. Die Autoren stellen auch die verschiedenen Neuentwicklungen dar, die Eingang in Zulassungsstudien beim Stadium III gefunden haben und deren finale Ergebnisse man bereits in diesem Jahr erwartet. Sie könnten ggf. die Therapiestandards ändern.

Bei rund 3000 Melanompatienten wird jährlich eine fortgeschrittene Metastasierung ihrer Erkrankung diagnostiziert, deren Verlauf in der Vergangenheit weitgehend unbeeinflussbar war. Innovationen aus der Molekularbiologie und Tumorimmunologie lassen nach mehr als $3 \mathrm{Jahr}$ zehnten ohne therapeutische Fortschritte beim metastasierten Melanom jetzt berechtigt auf eine Trendwende hoffen.

D. Schadendorf und H. Bernhard fassen die Entwicklung der neuen zielgerichteten Therapeutika, v. a. der selektiven BRAFund MEK-Inhibitoren ebenso wie die der Immunokologika zusammen, die aktuell zu einer Reihe von Neuzulassungen geführt hat. Diese Entwicklung zielt weiterhin auf die Erprobung von weiteren Medikamenten sowie auch deren Kombinationen. Studien stellen daher trotz der erzielten Fortschritte nach wie vor die beste Möglichkeit dar, die Behandlung des metastasierten Melanoms zu verbessern und betroffenen Patienten innovative und erfolgversprechende Behandlungsangebote zu machen.

Vor allem auch die Kombination der neu zur Verfügung stehenden Medikamente wird zur weiteren Prognoseverbesserung beitragen und ein Schwerpunkt der Therapieoptimierung beim metastasierten Melanom in den nächsten Jahren werden. Für die geeignete Auswahl individuell erfolgversprechender Medikamente wird dabei auch die vertiefte Genom- und Transkriptomsequenzierung von Tumorbiopsaten der jeweiligen Patienten beitragen können. Davon unabhängig wird der Erfolg einer Behandlung auch weiterhin durch interdisziplinäre Zusammenarbeit geprägt sein. Dies wird im Beitrag von $O$. Kölbl und F. Meier exemplarisch für die Therapie von Hirnmetastasen des Melanoms klar herausgestellt.

Zusammenfassend gilt, dass eine leitliniengerechte und interdisziplinäre multimodale Therapie des malignen Melanoms außerordentliche Bedeutung hat und noch kein Melanompatient im Stadium IV der Erkrankung in der Regel geheilt werden kann. Daher sollte mit dem Patienten besprochen werden, ob eine prioritäre Studienteilnahme möglich ist. Denn die augenblicklich in Studien verfügbaren Substanzen und Konzepte lassen weiterhin erwarten, dass die in Erprobung befindlichen Ansätze weitere (deutliche) klinische Fortschritte erzielen werden, die der Einzelanwendung der neu zugelassenen Substanzen alleine vermutlich überlegen sind.

Für die Schriftleiter des Schwerpunktthemas

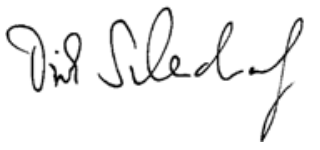

D. Schadendorf

Für die Herausgeber

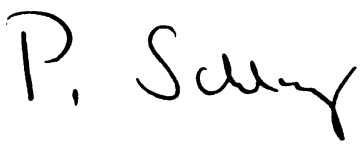

P.M. Schlag 


\section{Hier steht eine Anzeige.}

第 Springer 


\section{Korrespondenzadresse}

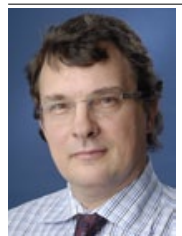

Prof. Dr. D. Schadendorf

Klinik für Dermatologie,

Universitätsklinikum Essen

Universität Duisburg-Essen

Hufelandstraße 50,

45122 Essen

dirk.schadendorf@uk-essen.de

\section{Einhaltung ethischer Richtlinien}

Interessenkonflikt. D. Schadendorf und P.M. Schlag geben an, dass kein Interessenkonflikt besteht.

\section{Literatur}

1. Pflugfelder A, Kochs C, Garbe C, Schadendorf D (2013) Evidenz-basierte S3-Leitline „Diagnose, Therapie und Follow-up des Melanoms". JDDG 11 (Suppl 6):1-116

2. www.awmf.org/leitlinien/detail/l//032-0240L.htm

\section{Reinhard J. Topf (Hrsg.) \\ Das krebskranke Kind und sein Umfeld}

Psychosoziale Aspekte der Versorgung und Unterstützung

Wien: new academic press 2014, 1., $346 \mathrm{~S}$., (ISBN 978-3-7003-1865-1), Softcover, 28.00 EUR

Die medizinische Versorgung von Kindern mit bösartigen Erkrankungen durch Chemotherapie, Strahlentherapie und chirurgische Maßnahmen ist die eine Sache. Eine andere ist die psychosoziale Begleitung von Kinder und Familien mit derartigen Erkrankungen. Beide Bereiche sind untrennbar miteinander verknüpft.

Reinhard Topf, Leiter der psychosozialen Dienste am St. Anna Kinderspital in Wien, dem größten hämatoonkologischen Behandlungszentrum Österreichs, hat nun seine Erfahrungen aus 25-jähriger Tätigkeit in diesem Bereich zusammengefasst. Dabei hat er einige frühere Publikationen um zusätzliche Themenfelder erweitert und gibt nun eine sehr umfassende Darstellung über „Das krebskranke Kind und sein Umfeld".

In 19 Kapiteln schreiben der Herausgeber und 25 Koautorinnen und -autoren über ihre Erfahrungen in den einzelnen Spezialgebieten. Dabei werden grundsätzliche Aspekte wie der Kinderschutz sowie dessen historische Entwicklung und das ganzheitliche psychosoziale Betreuungskonzept ebenso angesprochen wie einzelne spezialisierte Bereiche, beispielsweise die liäsonpsychiatrische Betreuung. Naturgemäß steht der psychosoziale Aspekt im Vordergrund. Medizinische Krebsaufklärung, psychosoziale Begleitung und Krankenhausseelsorge werden ebenso beschrieben wie die „Mutter als Leibärztin“. In weiteren Kapiteln wird die Bedeutung in Kindergarten, Schule, Spielgruppen und Kunsttherapie auch im Krankenhaus dargestellt.

Schließlich wird auch das Thema "Tod" und die Palliativbetreuung angesprochen. Für die - erfreulicherweise immer mehr - Überlebenden und deren Eltern ist natürlich das Thema "Lebensqualität" von entscheidender Bedeutung - auch dieser Aspekt wird in diesem Buch adäquat thematisiert. Das insgesamt 346 Seiten umfassende Werk schließt mit dem Kapitel „Rehabilitation“ und stellt die Bedeutung der "familienorientierten Rehabilitation" nach Krebserkrankungen dar. Leider ist es dem Autor und seinen Mitstreitern in jahrelanger Arbeit durch die Resistenz öffentlicher Institutionen noch immer nicht gelungen, diese in Österreich adäquat umzusetzen. Das im (Versand-) Buchhandel für 28 Euro beziehbare Werk kann all jenen empfohlen werden, die sich mit hämatoonkologischen Erkrankungen im Kindes- und Jugendalter beschäftigen. Es kann aber auch all jenen hilfreich sein, die in ihrer täglichen Arbeit Kinder und Jugendliche mit anderen schweren $\mathrm{Er}$ krankungen betreuen.

R. Kerbl (Leoben) 